\title{
AIP
}

\section{Affect of annealing on uniform and nonuniform strains in a sputtered Mo film on Si}

T. Adler and C. R. Houska

Citation: Journal of Applied Physics 50, 3288 (1979); doi: 10.1063/1.326369

View online: http://dx.doi.org/10.1063/1.326369

View Table of Contents: http://scitation.aip.org/content/aip/journal/jap/50/5?ver=pdfcov

Published by the AIP Publishing

\section{Articles you may be interested in}

Variation in stress with background pressure in sputtered Mo/Si multilayer films

J. Appl. Phys. 78, 2423 (1995); 10.1063/1.360164

Stress, strain, and microstructure of sputter-deposited Mo thin films

J. Appl. Phys. 70, 4301 (1991); 10.1063/1.349108

Effects of annealing on resistivity and on Schottky barrier heights of sputter-deposited MoSi2 films

J. Appl. Phys. 66, 4818 (1989); 10.1063/1.343796

As+ implantation and transient annealing of MoSi2 thin films

J. Vac. Sci. Technol. A 3, 718 (1985); 10.1116/1.573293

Plasma etching characteristics of sputtered MoSi2 films

Appl. Phys. Lett. 37, 466 (1980); 10.1063/1.91967

MIT LINCOLN

LABORATORY CAREERS

Discover the satisfaction of innovation and service

to the nation
- Space Control

- Air \& Missile Defense

- Communications Systems \& Cyber Security

- Intelligence, Surveillance and Reconnaissance Systems

- Advanced
Electronics
- Tactical Systems
- Homeland
Protection
- Air Traffic Control

- Air Traffic Control

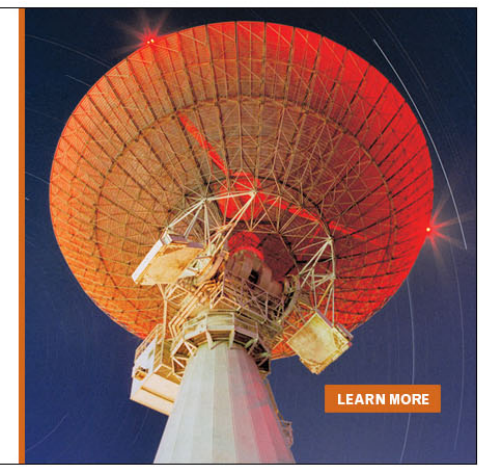




\title{
Affect of annealing on uniform and nonuniform strains in a sputtered Mo film on $\mathrm{Si}$
}

\author{
T. Adler and C. R. Houska \\ Department of Materials Engineering, Virginia Polytechnic Institute and State University, Blacksburg, \\ Virginia 24061
}

Sputtered films of $1.5 \mu \mathrm{m}$ of Mo deposited on (111)-oriented Si failed either by blistering or localized eruptions after various thermal treatments. Investigations were carried out to determine the amount of strain in the film associated with this unstable mechanical behavior. Two types of measurements were employed. One employs macroscopic interferometer measurements to measure deflection and the other $x$-ray diffraction. A separation is made of intrinsic and thermally induced strains. The intrinsic strains are believed to be due mainly to Ar atoms embedded during sputtering which remain throughout annealing treatments. These atoms also introduce a broadening of the diffraction lines because of the special constraints associated within films. This effect is separated from the usual line broadening due to dislocations and small particle size. Annealing treatments reconfirm that dislocation mechanisms are not as effective in relieving nonuniform microstrain in films as they are in cold-work filings of the same material.

PACS numbers: $61.70 .-\mathrm{r}, 81.15 . \mathrm{Cd}$

\section{INTRODUCTION}

During the process of examining interface reactions between micron-thick Mo and Ti films on Si single crystals, it was discovered that a parallel investigation was required to determine the amount of strain in the metal film. After annealing sputtered films within certain temperature ranges and quenching, the films failed either by blistering or localized eruptions. In each case, the kinetic study had to be discontinued.

It is well know that films contain high defect densities. In particular, defect densities of $10^{10}$ to $2 \times 10^{11}$ have been reported in $\mathrm{Cu}$ films ${ }^{1}$ which are of the same magnitude as densities found in the cold-worked state. On annealing, the strains locked into films are less responsive to thermal treatment than cold-worked filings. ${ }^{2,3}$ Also, stresses in the plane of a film as large as $10^{-3} \mu$ to $10^{-2} \mu$ ( $\mu$ is the shear modulus) have been observed. ${ }^{4}$ This may be compared with a flow stress of $10^{-5} \mu$ to $10^{-4} \mu$ for bulk single-crystal specimens. In films, dislocation mechanisms cannot effectively relieve stresses since dislocation motion is either impeded or their generation by sources is restricted. Besides grain boundaries or subboundaries acting as obstacles to dislocation motion, the interface barrier may not allow dislocations to cross. This could provide considerable stress build-up at interfaces containing intermediate brittle films formed on annealing.

In modeling the elastic problem between a film and substrate, ${ }^{5}$ the following assumptions are made: (1) The film is firmly attached to the substrate. (2) The thermal expansion of the film and substrate are isotropic in the plane of the substrate. (3) The stress on the film in a direction normal to the plane of the film is zero. (4) The shear strain in the plane of the film is zero. It is assumed that a biaxial strain model closely approximates the experimental conditions.
If the adhesion between film and substrate were ideal, more strain at the film/substrate interface is expected than at the film surface. ${ }^{6}$ This departure from biaxial strain requires a triaxial elastic model. The nonuniformity of strain perpendicular to $\mathrm{a} \mathrm{Pb}$ film has been studied' at two temperatures for three times by $\mathrm{x}$-ray diffraction.

Except for the preceding $x$-ray study by Murakami, all other $x$-ray investigations have made use of conventional line-broadening methods originally developed to characterize cold-worked metals. ${ }^{8}$ This may or may not be adequate depending upon the materials, the method of preparation, and the subsequent thermomechanical history. For sputtered samples, only a portion of the line broadening resembles that found in cold-worked metals, while another component is related to pointlike defects which are found in the special elastic environment of a polycrystalline film. Although point defects are not expected to produce line broadening in unconstrained grains, line broadening can be produced under the special constraints found in sputtered films. This paper considers both forms of broadening.

\section{THEORETICAL BACKGROUND}

The development of internal strains within sputtered films may be simplified into two stages of the initial specimen preparation. The continuous deposition of a film layer by layer from moderately energetic atoms can build in special strains related to embedded point defects, as well as strains resulting from subsequent temperature changes.

Figure 1 illustrates such a sequence. Figure 1(a) schematically represents the interaction of point defects (see rectangles) with fixed subboundaries putting the surrounding matrix in compression along the $X$ direction [Fig. 1(b)]. A resultant Poisson expansion is shown along $Z$ while Figs. 


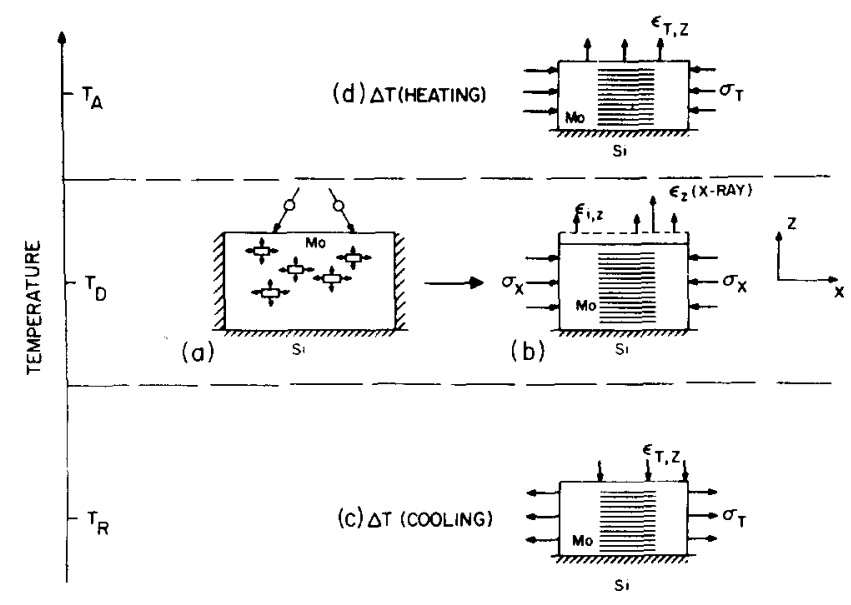

FIG. 1. Schematic representations of (a) interaction of point defects with fixed subboundaries, (b) resultant compressive stress, $\sigma_{x}$, and Poisson expansion, $\epsilon_{i, Z}$, at the deposition temperature, $T_{D}$, (c) Poisson strain, $\epsilon_{T, Z}$, developed on cooling to room temperature, $T_{R}$, (d) Poisson strain, $\epsilon_{T, Z}$, developed on heating above the deposition temperature, $T_{A}$.

$1(c)$ and 1(d) isolate the effect of temperature changes for a film with a greater thermal expansion coefficient than its substrate. The diagrams also illustrate an unconstrained displacement of the free surface. In the present study, the surface receives Mo ions and an occasional Ar ion at high velocities $\left(\sim 10^{3}-10^{6} \mathrm{~cm} \mathrm{~s}^{-1}\right)$. These may stick to the surface, bounce off, or be driven into film already deposited. If Mo is driven into an interstitial site or the relatively large Ar atoms become embedded within the lattice, point defects are generated which may or may not be able to rearrange into more complex arrangements as a result of their mutual elastic interaction. Normally, one considers the point defect to be in an unconstrained lattice with all surfaces free to move. This is not the case for adherent sputtered films except at the free surface which can move along the $Z$ direction.

On cooling to room temperature [Fig. 1(c)], the Mo film will attempt to contract more than the Si substrate. ${ }^{9}$ This resultant thermal stress, $\sigma_{T, x}$, combines with the intrinsic stress, $\sigma_{i, x}$, giving a deflection $Z$ of the disk-shaped sample which is proportional to the resultant film stress $\sigma_{x}$. Using isotropic elasticity, these superimpose as follows:

$$
\sigma_{x}=\sigma_{i, x}+\sigma_{T}=\frac{E}{3(1-v)} \frac{Z_{s}^{2}}{Z_{f}} \frac{Z}{X^{2}},
$$

where $E$ and $v$ are Young's modulus and Poisson's ratio for the Si substrate $\left[E /(1-v)=2.305 \times 10^{12} \mathrm{dyn} / \mathrm{cm}^{2}{ }^{10}\right], Z_{s}$ and $Z_{f}$ are the substrate and film thicknesses, and $Z$ is the deflection at a distance $X$ from the center of the circular disk. [See Fig. 1(b) for $X$ and $Z$ directions.] Also, the deflection is obtained by counting optical fringes $n$ in an interference diagram with

$$
Z=\frac{1}{2} n \lambda,
$$

and $\lambda=5.461 \AA$ for a $\mathrm{Hg}$ vapor light source. The thermal stress generated in the Mo film on cooling from the deposition temperature $\left(T_{D}\right)$ to room temperature $\left(T_{R}\right)$ depends on the thermal expansion coefficients $\alpha_{\mathrm{Mo}}$ and $\alpha_{\mathrm{Si}}$ and the elastic constants $E_{\mathrm{Mo}}$ and $v_{\mathrm{Mo}}$.

$$
\sigma_{T}=\left(\alpha_{\mathrm{Mo}}-\alpha_{\mathrm{Si}}\right)\left(T_{D}-T_{R}\right) \frac{E_{\mathrm{Mo}}}{1-v_{\mathrm{Mo}}} .
$$

Also, the Poisson strain along $Z$ is

$$
\epsilon_{T, Z}=-\left(\alpha_{\mathrm{Mo}}-\alpha_{\mathrm{Si}}\right)\left(T_{D}-T_{R}\right) \frac{2 v_{\mathrm{Mo}}}{1-v_{\mathrm{Mo}}} .
$$

Since $\alpha_{\mathrm{Mo}}>\alpha_{\mathrm{Si}}$ and if $\sigma_{i, X}=0$ at the deposition temperature, the film would be in tension on cooling to room temperature. However, the inclusion of an intrinsic stress can reduce or dominate $\sigma_{T}$ such that the film goes into compression. On heating above $T_{D}$, thermal and intrinsic stresses add in compression.

The stress-related Poisson strain perpendicular to the interface is of interest when comparing the preceding interferometer data with $\mathbf{x}$-ray results. This is given by

$$
\begin{aligned}
\epsilon_{Z} & =\epsilon_{i, Z}+\epsilon_{T, Z} \\
& =-\frac{E}{3(1-v)}\left(\frac{2 v_{\text {Mo }}}{E_{\text {Mo }}}\right) \frac{Z_{S}^{2}}{Z_{f}} \frac{Z}{X^{2}}
\end{aligned}
$$

with $v_{\mathrm{Mo}}=0.32$ and $E_{\mathrm{Mo}}=3.2 \times 10^{12} \mathrm{dyn} / \mathrm{cm}^{2}$. A more rigorous calculation ${ }^{5}$ has been carried out for $\epsilon_{T, Z}$ rather than simply multiplying $\sigma_{T}$ by $2 v_{\mathrm{Mo}} / E_{\mathrm{Mo}}$. However, for Mo films, the discrepency is only $4 \%$ for the (110) planes and, therefore, the isotropic calculation is used. The strain, $\epsilon_{z}$, influences those atomic planes which are parallel to the free surface and normally measured from conventional $2 \theta$ scans with a diffractometer. The $x$-ray strain data need not agree with the interferometer data. An additional dilation $\epsilon_{X R, Z}=\Delta Z / Z$ from the unconstrained movement of the free surface due to embedded atoms must be included in the $\mathrm{x}$-ray measurement. The fractional change in the spacing is obtained from

$$
\epsilon_{Z}^{\prime}=\epsilon_{i, Z}+\epsilon_{X R, Z}+\epsilon_{T, Z}=\frac{1}{2}\left(\cot \theta_{B}\right) \Delta 2 \theta,
$$

where $\epsilon_{Z}^{\prime}$ distinguishes the $\mathrm{x}$-ray determination of strain along the $Z$ direction, and $\Delta 2 \theta=2 \theta_{B}-2 \theta_{\text {Mo }}$ which is the angular line shift relative to unstrained Mo powder.

In a previous paper, ${ }^{11}$ it was shown that the state of nonuniform microstrain can be described by the convolution of two Gaussian strain distributions. One represents the strain distribution from an average dislocation network, while the other describes local variations in the uniform column strain. The latter can be related to local variations in the strain produced by embedded atoms. In this case, one allows the uniform strain within a column of unit cells perpendicular to the plane of reflection to vary between grains. This information is obtained from analysis of line shapes by fitting the following Fourier series over two orders $(l=1,2) .{ }^{11}$

$$
\begin{aligned}
P^{\prime}(2 \theta)= & \frac{K N F^{2}}{\sin ^{2} \theta_{B}}\left(1+2 \sum_{n=1}^{3 / 2 N_{1}}\left(A^{I}\right)^{n} A_{n}^{S}\left(A_{1}^{D}\right)^{n}\right. \\
& \left.\times\left(A_{1}^{U}\right)^{n^{2}} \cos \left[2 \pi\left(h_{3}-l\right)\right]\right)
\end{aligned}
$$

where $K$ is taken to be a constant, $N$ is the total number of 


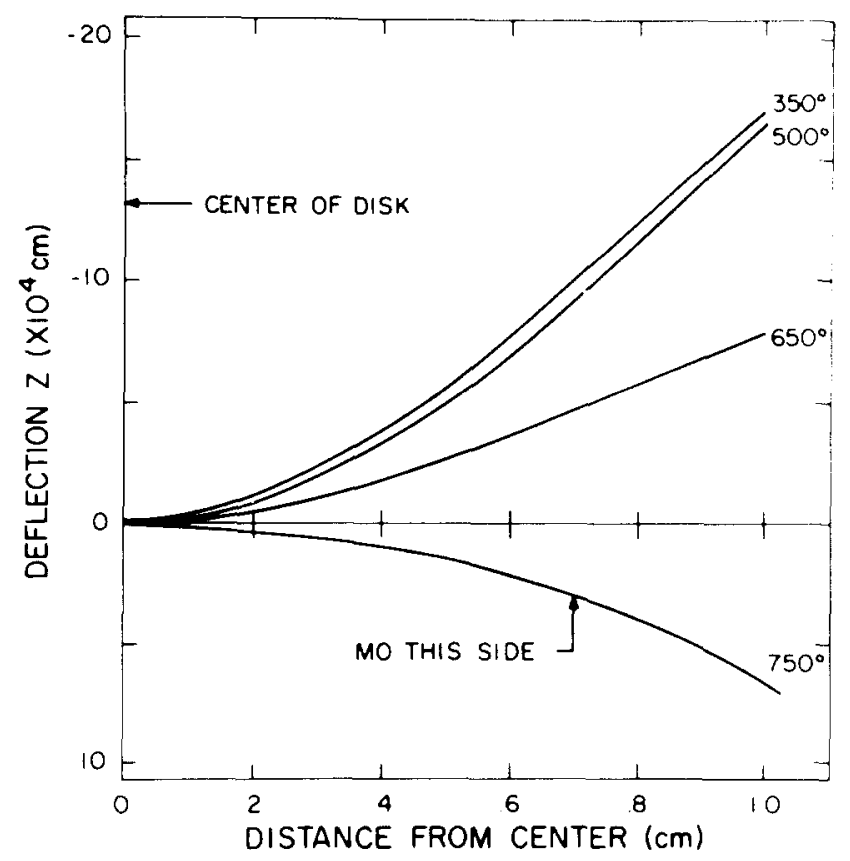

FIG. 2. Deflection data for Mo/Si disks obtained from interference microscopy.

unit cells, $F^{2}$ is the structure factor squared, $\theta_{B}$ is the Bragg angle, $A^{I}$ is the instrumental coefficient,

$$
A_{n}^{S}=1-\frac{|n|}{N_{3}}+\frac{4}{27}\left(\frac{|n|}{N_{3}}\right)^{3} \quad \text { for }|n|<\frac{3}{2} N_{3}
$$

and

$$
\begin{aligned}
& A_{n}=0 \quad \text { for }|n| \geqslant \frac{3}{2} N_{3}, \\
& \left(A_{1}^{D}\right)^{n}\left(A_{1}^{U}\right)^{n^{2}}=\exp \left[-2 \pi^{2}\left(\frac{\left\langle\epsilon_{1 D}^{2}\right\rangle}{n}+\left\langle\epsilon_{1 U}^{2}\right\rangle\right) n^{2} l\right],
\end{aligned}
$$

and

$$
h_{3}=2\langle d\rangle \sin \theta / \lambda
$$

with $\langle d\rangle$ the average interplanar spacing. The quantities that are of special interest are $N_{3}\langle d\rangle$ the average subgrain size perpendicular to the reflecting planes, $\left\langle\epsilon_{I D}^{2}\right\rangle$ and $\left\langle\epsilon_{I U}^{2}\right\rangle$ are mean square strains due to dislocations and embedded atoms, respectively.

\section{EXPERIMENTAL RESULTS}

Molybdenum films $1.5 \mu \mathrm{m}$ thick were deposited onto polished $\frac{7}{8}$-diam Si substrates with the 111 planes oriented to within $\pm 0.5^{\circ}$ of the free surface. The substrates were chemically cleaned and then sputter etched at $0.8 \mathrm{kV}, 60 \mathrm{~W}$ for $30 \mathrm{~min}$ to remove the oxide. Without exposing the cleaned surface to the atmosphere, a $1.5-\mu \mathrm{m}$ film of Mo was sputtered with the target at $2.8 \mathrm{kV}$ and a power of $450 \mathrm{~W}$ for $41.2 \mathrm{~min}$ using an Ar pressure of $5 \mu \mathrm{m}$. The Mo target was 99.95\% pure. Also, the substrate temperature during deposition was between 340 and $360^{\circ} \mathrm{C}$. X-ray spectroscopy revealed a small amount of $\mathrm{Ar}$ to be present which remained even after annealing at $750^{\circ} \mathrm{C}$.

A set of samples were annealed in the range $950-970^{\circ} \mathrm{C}$ under vacuum using an ion pump which was backed up with a sorption pump. On cooling to room temperature, the entire Mo film blistered. Both 15- and 30-min anneals resulted in blistered films on cooling to room temperature. Other experiments demonstrated that this is independent of cooling rate. Because of the strong tendency for reacted $1.5-\mu \mathrm{m} \mathrm{Mo}$ films to fail after thermal treatments in the range 900 $1000{ }^{\circ} \mathrm{C}$, the reaction temperature was reduced in order to carry out studies at room temperature on quenched samples. The other obvious alternative is to investigate film behavior at temperature realizing that a reacted $1.5-\mu \mathrm{m}$ Mo film may become mechanically unstable on cooling to room temperature.

A series of step anneals held for $2 \mathrm{~h}$ at $350,500,650$, and $750^{\circ} \mathrm{C}$ on a single sample were carried out. After the last anneal at $750^{\circ} \mathrm{C}$ an $\mathrm{x}$-ray examination revealed that the sample was still largely pure Mo and $\mathrm{Si}$. However, 9 weak lines could be attributed to a small amount of hexagonal $\mathrm{MoSi}_{2}$ and 14 weak lines were attributed to $\mathrm{Mo}_{5} \mathrm{Si}_{3}$. These became observable after the $750^{\circ} \mathrm{C}$ anneal. Lines of intermediate intensity which could not be identified were present throughout the step anneal and systematically increased in intensity and in number as the sample was taken to higher temperatures. Also, their room-temperature positions shifted systematically toward a higher angle as the reaction temperature was raised.

Figure 2 illustrates the shape changes of the circular disks with step annealing. Initially, the bending was toward a center on the substrate side putting the Mo film in compression. No change in curvature occured after annealing at the deposition temperature $\left(350^{\circ} \mathrm{C}\right)$ for $2 \mathrm{~h}$ and very little change occurred at $500^{\circ} \mathrm{C}$. Large changes in curvature were observed at 650 and $750^{\circ} \mathrm{C}$. In fact, the $750^{\circ} \mathrm{C}$ anneal caused the film to blister and go into tension.

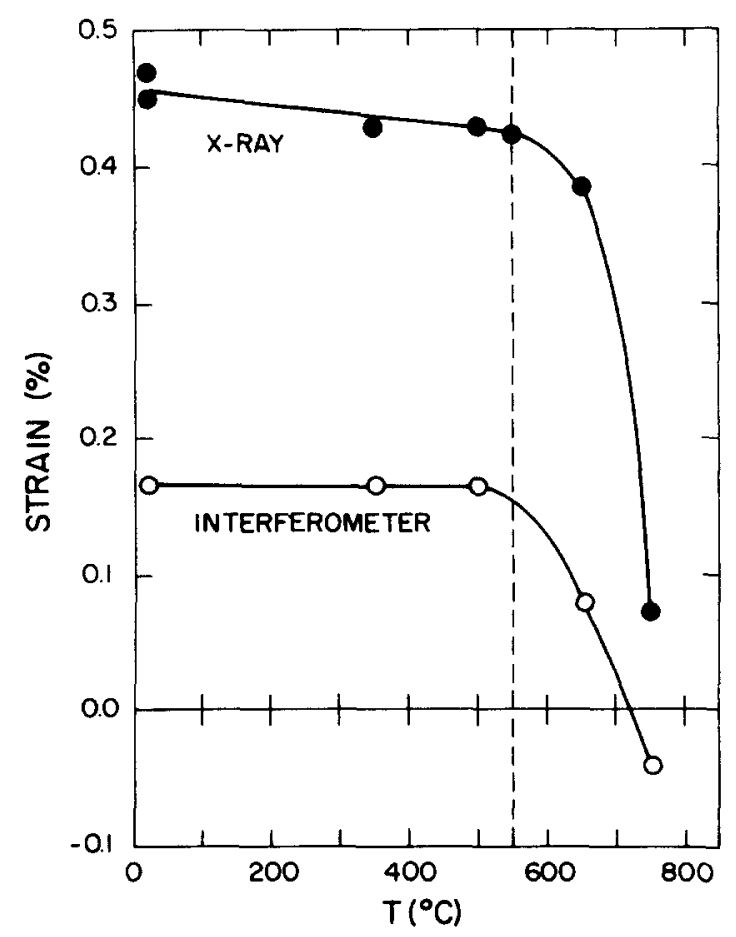

FIG. 3. Strain perpendicular to sample surface obtained from interference microscopy, $\epsilon_{Z}$, and from x-ray diffraction, $\epsilon_{Z}^{\prime}$. 


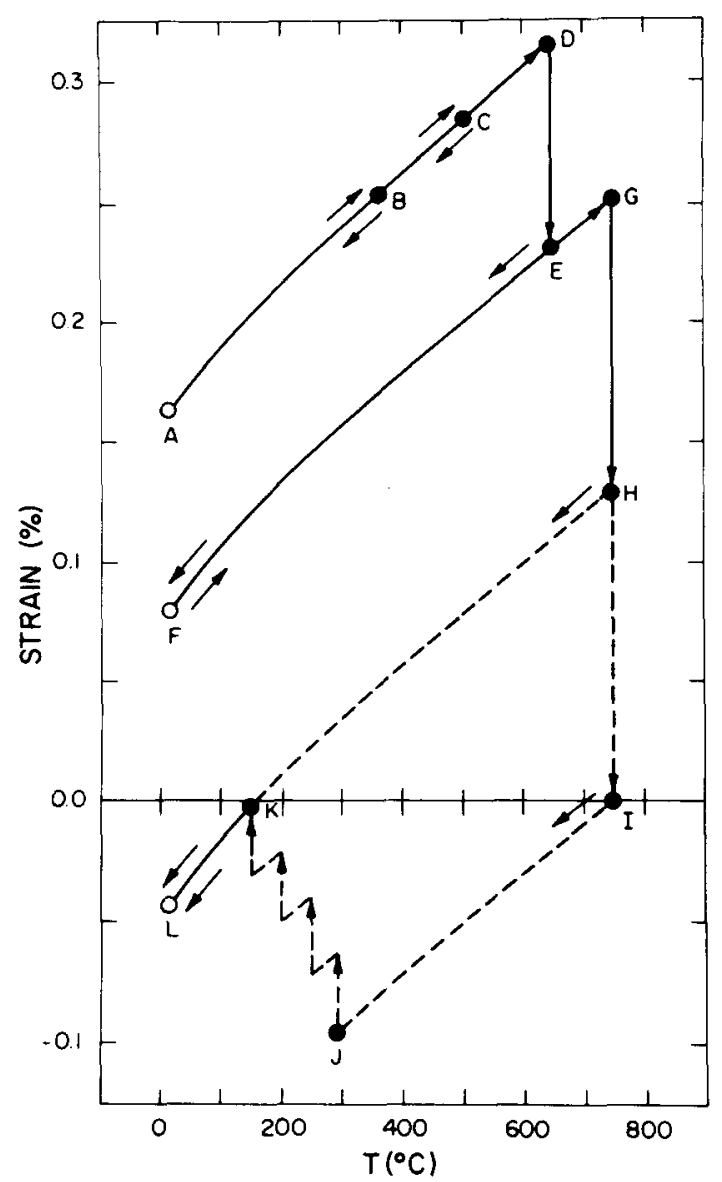

FIG. 4. Hypothetical strain, $\epsilon_{Z}$, as a function of cooling and heating cycles calculated from data points $A, F$, and $L$ and thermal expansion data (see Ref. 9).

Figure 3 is a plot of $\epsilon_{Z}$ and $\epsilon_{Z}^{\prime}$ determined from Eqs. (4) and (5) using the inner rings of the interference patterns. This again illustrates the shape change from convex to concave disks going from film compression to tension over the temperature range $600-750^{\circ} \mathrm{C}$.

Figure 4 illustrates the variation in thermal strain obtain from Eq. (3'). Point $A$ represents the strain in the asreceived Mo film. Heating to 350 (point B) and $550^{\circ} \mathrm{C}$ produces no signigicant relaxation. However, heating to $\mathrm{D}$ results in a relaxation of about $0.1 \%$. The curve A-D-E-F was drawn to illustrate creep at $650^{\circ} \mathrm{C}$. The last path beginning at $\mathrm{F}$ and ending at $\mathrm{L}$ is uncertain because of blistering on cooling. Large local plastic deformations occured in the Mo at the blisters, causing local decreases in $\epsilon_{Z}$. The end point $\mathrm{L}$ could be reached either by creep of about $0.13 \%$ at $750{ }^{\circ} \mathrm{C}$ or by complete stress relaxation by creep at $750^{\circ} \mathrm{C}(\mathrm{E}$ to $\mathrm{I})$ and blistering when the sample reaches a critical tensile strain $\mathrm{J}$. These represent extreme possibilities and the true behavior is likely to be intermediate with creep occurring to a point between $\mathrm{H}$ and $\mathrm{I}$. Studies are in progress to better determine the strain relaxation at temperature.

Figure 5 contains half-widths from four $x$-ray lines measured at room temperature after a $2 \mathrm{~h}$ step annealing. These increase continuously with increasing $2 \theta$ and drop sharply after annealing at 650 and $750^{\circ} \mathrm{C}$. The sharpening of all lines in this range is due to a decrease in nonuniform microstrain and an increase in $x$-ray particle size.

A more detailed x-ray study was carried out at $550^{\circ} \mathrm{C}$ which corresponds to the transition temperature for the $2 \mathrm{~h}$ step anneals. As the temperature is increased above $550^{\circ} \mathrm{C}$, strain relaxation and the formation of silicides proceed at an increasing rate. At $550^{\circ} \mathrm{C}$, annealing up to $192 \mathrm{~h}$ does not produce significant orientation changes within the Mo film, i.e, the relative line intensities remained constant. The intensities and number of the unidentified lines increased; however, neither $\mathrm{MoSi}_{2}$ or $\mathrm{Mo}_{5} \mathrm{Si}_{3}$ were formed in sufficient amounts to give measurable diffraction lines. After $192 \mathrm{~h}$ of annealing, the thickness of Mo decreased slightly. The decrease in $\epsilon_{Z}^{\prime}$ after $27 \mathrm{~h}$ was found to be $8 \%$. As expected, long-term annealing at $550^{\circ} \mathrm{C}$ can result in creep at a lower temperature than what was found for the $2 \mathrm{~h}$ step anneals. In this case, the increase in time is a factor of about 100 when the temperature is dropped from 650 to $550^{\circ} \mathrm{C}$.

Results of the line-shape analysis after isothermal annealing at $550{ }^{\circ} \mathrm{C}$ are given in Table I. Two methods of analyzing the line-shape data give equally good fits. " The first gives an extrapolated first neighbor strain $\left\langle\epsilon_{1}^{2}\right\rangle^{1 / 2}$ and an exponential parameter $r$ which allow the rms microstrain between cells to be determined from

$$
\left\langle\epsilon_{n}^{2}\right\rangle^{1 / 2}=|n|^{r}\left\langle\epsilon_{1}^{2}\right\rangle^{1 / 2} \text {. }
$$

In this representation of strain, one considers each diffracting grain to be made up of columns of cells arranged perpendicular to the reflecting planes (or free surface). Equation (7) represents the rms strain between all $n$th neighbor cells averaged over all columns. A pair of cells may not cross over columns. If $r$ values, obtained from sputtered and annealed Mo films, are compared with those obtained from annealed Mo filings, one finds the film values to be about one-half the value obtained from filings. This gives a much slower fall off of strain with distance for the Mo film. A value close to

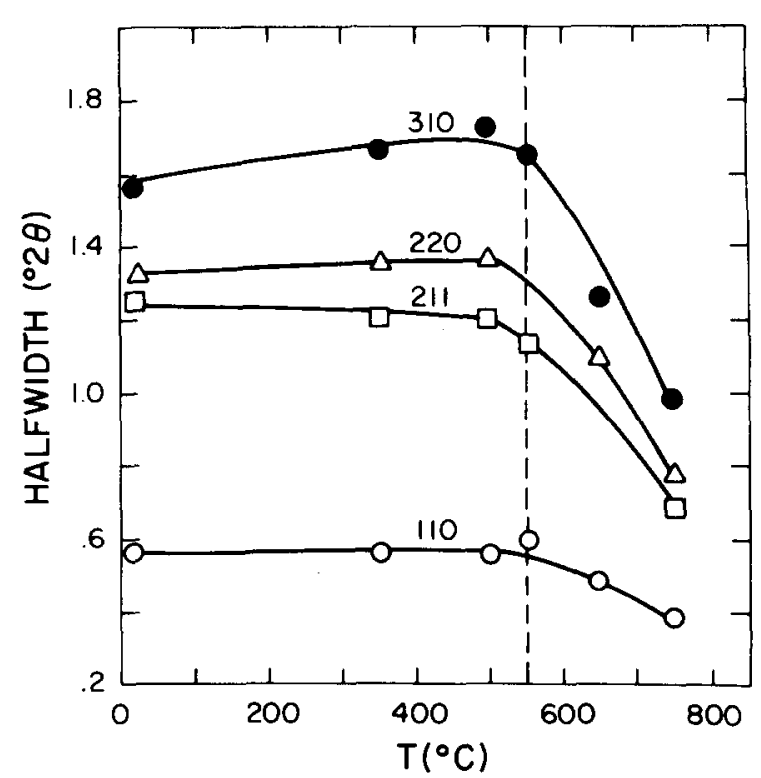

FIG. 5. X-ray half-width data from sputtered Mo film on a (111)-oriented Si substrate. 
TABLE I. Particle size $\left(N_{3}\langle d\rangle\right)$ extrapolated first neighbor rms strain parameters $\left(\left\langle\epsilon_{1}^{2}\right\rangle^{1 / 2}, r,\left\langle\epsilon_{1 D}^{2}\right\rangle^{1 / 2}\right.$, and $\left.\left\langle\epsilon_{1 U}^{2}\right\rangle^{1 / 2}\right)$, for $(110)$ planes from a Mo film annealed at $550^{\circ} \mathrm{C}$. Data from as-filled and annealed Mo powders are also given for comparison

\begin{tabular}{lllllll}
\hline \hline Time $(\mathrm{h})$ & $N_{3}$ & $N\langle d\rangle(\mathrm{A})$ & $\left\langle\epsilon_{1}^{2}\right\rangle^{1 / 2}$ & $r$ & $\left\langle\epsilon_{1 D}^{2}\right\rangle^{1 / 2}$ & $\left\langle\epsilon_{1 \ell}^{2}\right\rangle^{1 / 2}$ \\
\hline 0 & 87 & 194 & 0.0118 & -0.272 & 0.0177 & 0.0029 \\
27 & 97 & 215 & 0.0110 & -0.264 & 0.0167 & 0.0029 \\
192 & 125 & 278 & 0.0098 & -0.245 & 0.0151 & 0.0028 \\
As-filed & 117 & 260 & 0.0201 & -0.559 & & \\
$415^{\circ} \mathrm{C}(1 \mathrm{~h})$ & 173 & 385 & 0.0123 & -0.524 & & \\
$660^{\circ} \mathrm{C}(1 \mathrm{~h})$ & 222 & 495 & 0.00803 & -0.481 & & \\
\hline
\end{tabular}

$r=-\frac{1}{2}$ is usually found for cold-worked filings. ${ }^{12}$ This intermediate value for $r$ has been interpreted using a second method of analysis which requires a convolution of two distinct Gaussian strain distributions. One distribution requries $r=-\frac{1}{2}$ due to dislocation fields, while the second Gaussian with $r=0$ describes the distribution of uniform column strain due to fluctuations in the density of the embedded atoms. The standard deviation parameters for each distribution are given by the quantities $\left\langle\epsilon_{1 D}^{2}\right\rangle$ and $\left\langle\epsilon_{1 U}^{2}\right\rangle$.

The first neighbor rms strain parameter, $\left\langle\epsilon_{1 U}^{2}\right\rangle$ and $\left\langle\epsilon_{1 D}^{2}\right\rangle$, should only be interpreted in connection with Eq. (7) over the range $10 \leqslant n \leqslant N_{3}$. A typical line-profile analysis can give an accurate fit of Fourier coefficients over the peak portion of the curve and usually not the low-intensity tail portion of the profile which is sensitive to the low values of $n$. Considerable overlap of adjacent peaks for powders and film patterns excludes a meaningful investigation of this region and, therefore, of the near-neighbor displacement. The range $10 \leqslant n \leqslant N_{3}$ includes a large proportion of the atom pairs in the sample and is, therefore, of considerable interest. Furthermore, the range $n>N_{3}$ is likely to be a smooth continuation of the measured curve. An experimental determination of the Fourier coefficients with large $n$ requires a highly accurate determination of the instrumental broadening curve.

Table $I$ also includes a listing of the average particle size $\left(N_{3}\langle d\rangle\right)$ as well as the average number of cells per column $\left(N_{3}\right)$. The $\mathrm{x}$-ray particle size may be taken as an average separation between dislocations. This, of course, increases slowly with annealing time at $550^{\circ} \mathrm{C}$.

\section{DISCUSSION}

The quantities $\epsilon_{i, Z}, \epsilon_{X R . Z}$, and $\epsilon_{T, Z}$ can be determined using Eqs. (3'), (4), and (5). In the as-received sample at room temperature, $\epsilon_{i, Z}=0.255 \%, \epsilon_{X R, Z}=0.285 \%$, and $\epsilon_{T: Z}=-0.088 \%$. All of these quantities refer to dimensional changes of (110) planes along a line perpendicular to the $\mathrm{Mo} / \mathrm{Si}$ interface. It is evident that the intrinsic Poisson strain $\epsilon_{i, Z}$ dominates the thermal term. This introduces a compressive strain on cooling to room temperature. The term $\epsilon_{X R, Z}$ results from a free expansion of the lattice due to embedded atoms and does not change greatly with annealing below $650^{\circ} \mathrm{C}$. A decrease occurs after $2 \mathrm{~h}$ at $750^{\circ} \mathrm{C}$.

$\mathrm{X}$-ray spectroscopy reveals the presence of Ar after all step anneals including $750^{\circ} \mathrm{C}$. However, a $20 \%$ decrease of the $K_{\alpha}$ and $K_{\beta}$ lines of Ar was found at $750^{\circ} \mathrm{C}$. It should be realized that because of the high absorption of $\operatorname{Ar} K$ radi- ation in Mo only Ar near the free surface can be detected.

If one assumes that the lattice expansion is due to Ar atoms which substitute for Mo and the corresponding atomic radii are 1.92 and $1.47 \AA,^{13} 0.9 \%$ of Ar would be sufficient to give the measured value of $\epsilon_{X R, Z}=0.285 \%$. It is possible that this is an underestimate because Ar in the grain boundaries is not considered in the estimate. Also, it is assumed that the lattice expansion is due to Ar and not interstitial Mo. This would tend to compensate for Ar not detected at the grain boundaries. However, one would expect a greater response with annealing if interstitial Mo were the dominant cause of the lattice expansion. Consequently, interstitial Mo is not believed to be present in significant concentrations.

The variation in the rms strain with cell separation $n$ (in units of $\langle d\rangle$ ) can be seen in Fig. 6 for the as-received sample as well as for $192 \mathrm{~h}$ at $550^{\circ} \mathrm{C}$. The significance of $\left\langle\epsilon_{n}^{2}\right\rangle$ in relation to $\epsilon_{Z}^{\prime}$ may not be appreciated from this plot. The average strain, $\left\langle\epsilon_{n}^{2}\right\rangle$, represents the standard deviation for the Gaussian distribution as obtained from the $x$-ray lineshape analysis. When normalized and written in terms of an origin $\epsilon_{Z}^{\prime}$, this is given by

$$
P_{R}=\left(1 / 2 \pi\left\langle\epsilon_{n}^{2}\right\rangle\right)^{1 / 2} \exp \left[-\left(\epsilon_{n}-\epsilon_{Z}^{\prime}\right)^{2} /\left(2\left\langle\epsilon_{n}^{2}\right\rangle\right)\right] .
$$

Figure 7 illustrates distributions for cell specings of $n=10$, 30 , and 50 in the as-prepared sample. For $n=10,24 \%$ of the

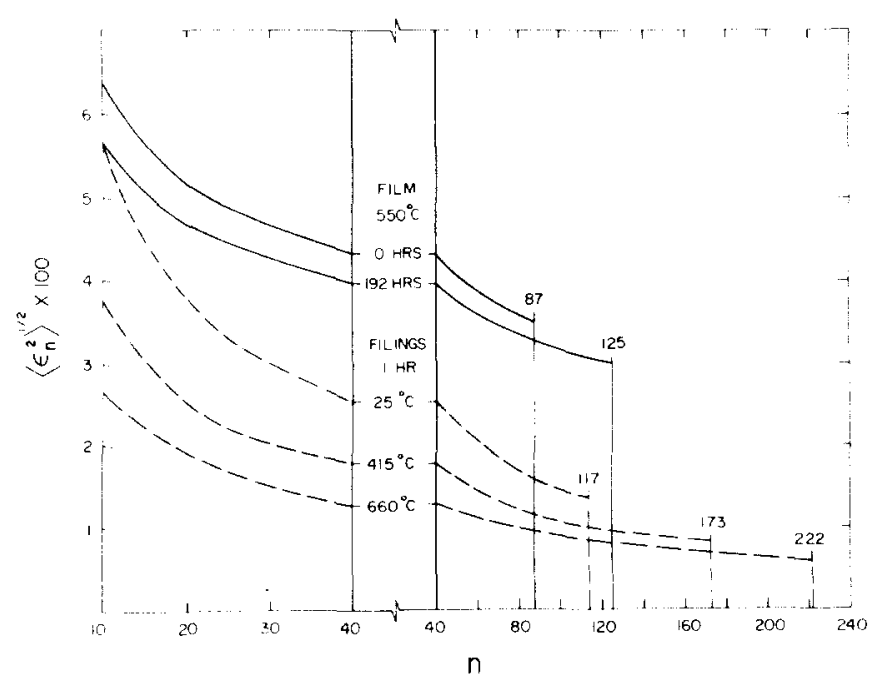

FIG. 6. Variation in rms microstrain along [110] with cell separation for a sputtered Mo film on a (111)-oriented Si substrate in the annealed and asreceived conditions. Corresponding strain for Mo filings and after the indicated anneals (see Ref. 14) 


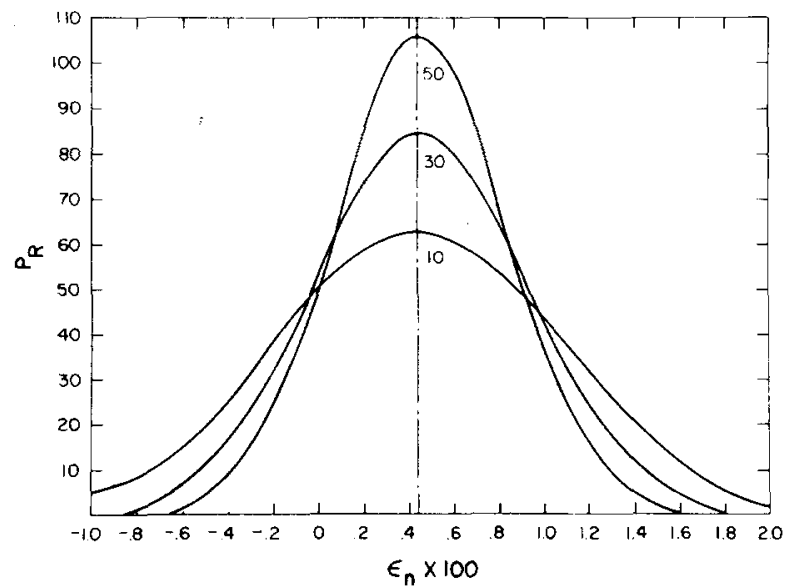

FIG. 7. Normalized strain distributions $\left(\int P_{R} d \epsilon_{Z}=1\right)$ for the as-prepared $\mathrm{Mo} / \mathrm{Si}$ sample at $n=10,30$, and 50 .

distribution corresponds to a negative $\epsilon_{n}$, while for $n=30$ and 50 this reduces to approximately 17 and $12 \%$, respectively. In each case, the average strain along $Z$ within (110) columns is positive and negative along $X$. The results from $x-$ ray line-broadening studies which give $\left\langle\epsilon_{n}^{2}\right\rangle$ must be associated with the variation in strain about an average or uniform component of strain. Also, the decrease in $\left\langle\epsilon_{n}^{2}\right\rangle$ with $n$ is characteristic of cold-work materials containing high dislocation densities. The upper curves of Fig. 6 would both level off at about $0.29 \%$ strain which represents the contribution due to variations in the uniform column strain $\left(\left\langle\epsilon_{U}^{2}\right\rangle^{\frac{1}{2}}\right)$.

The lower curves of Fig. 6 provide a comparison with the annealing behavior of cold-worked Mo filings ${ }^{14}$ In this case the fall off of $\left\langle\epsilon_{n}^{2}\right\rangle^{\frac{1}{2}}$ with $n$ is characteristic of unconstrained cold-worked filings. Filings are seen to be more responsive to annealing. A large drop in the strain curve is evident between 415 and $660^{\circ} \mathrm{C}$ after only $1 \mathrm{~h}$ at temperature. Even though the Mo film was annealed at $550^{\circ} \mathrm{C}$ for $192 \mathrm{~h}$, the rms strain still remains well above the $415^{\circ} \mathrm{C} 1-\mathrm{h}$ curve of the filings. Likewise, one finds that the distribution of uniform strain decreases very little. These results are in accord with the conclusion that dislocation mechanisms cannot effectively relieve stresses in thin films. ${ }^{4}$

The origin of the uniform column strain and variations about the average is of considerable practical interest within sputtered films. As one might expect, it is absent in coldworked filings. The source of this strain might be expected to give well-known diffraction effects characteristic of point defects. ${ }^{15}$ If oversize point defects were present in an unconstrained grain, one would expect no line broadening but an increase in the lattice parameter and a small decrease in the intergrated intensitites of the low-angle lines used in this investigation. The lattice parameter does increase and line broadening is observed. An explanation of the latter requires a new solution to the elastic problem which contains special constraints relatable to immovable boundaries as well as one boundary which remains free to move. Without a quantitative solution, one must use qualitative arguments to explain the behavior of sputtered films. Fissures were observed on the film surface after $\mathrm{MoSi}_{2}$ was detected and with an unreacted Mo film still remaining. It is reasonable to find that film failure correlates with the observation of a hard brittle phase and a net uniform tension. A previous discussion, emphasized that large fluctuations of strain (or stress) about the average strain is usually observed especially at small distrances $n$. When the average strain is tensile, fluctuations could provide an additional increment of tensile strain which initiates crack formation or propagation.

In conclusion, this study deals mainly with one Mo film preparation on a Si substrate. It has been demonstrated that sputtered Mo may also be initially in tension; however, this is usually associated with entrapped oxygen. Argon has been shown to be present when film compression is found. ${ }^{10}$

\section{ACKNOWLEDGMENTS}

The authors are grateful to Professor C.B. Ling of the VPI and SU Mathematics Department for discussions of the elastic problems encountered in this reasearch. This research was made possible by funding from the National Science Foundation under contract No. DMR 75-17201.

'A. Gangulee, J. Appl. Phys. 43, 867 (1972).

${ }^{2}$ A. Gangulee, J. Appl. Phys. 43, 3943 (1972).

${ }^{3}$ A. Gangulee, J. Appl. Phys. 43, 177 (1974).

'P. Chaudhari, S. Mader, and J.F. Freedman, J. Vac. Sci. Technol. 6, 618 (1969).

'R.W. Vook and F. Witt, J. Appl. Phys. 36, 2169 (1965)

'P. Chaudhari, IBM J. Res. Dev. 13, 197 (1969).

M. Murakami, Acta Metall. 26, 175 (1978)

${ }^{8}$ B.E. Warren, Prog. Met. Phys. 8, 147 (1959)

${ }^{9}$ Thermophysical Properties of Matter (IFI/Plenum, New York, 1975)

${ }^{10}$ A.G. Blachman, Metall. Trans. 2, 699 (1971).

"T. Adler and C.R. Houska, preceding paper, J. Appl. Phys. 50, 3282 (1979)

${ }^{12}$ R.L. Rothman and J.B. Cohen, Advances in X-Ray Analysis, 12, edited by C.S. Barrett, J.B. Newkirk, and G.R. Mallett. (Plenum, New York, 1969).

${ }^{13}$ C.J. Smithells, Met. Ref. Book (Plenum, New York, 1967).

${ }^{14 J}$. Despujols and B.E. Warren, J. Appl. Phys. 28, 195 (1958).

'K. Huang, Proc. R. Soc. London A 90, 102 (1947). 